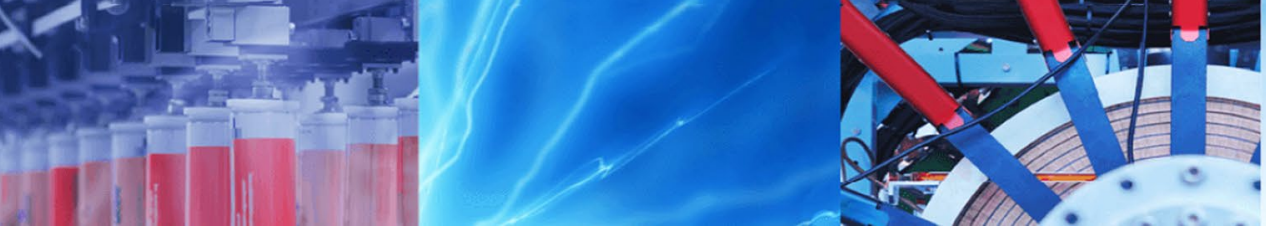

Research Article

\title{
Developing a fast and robust numerical method for the simulation of cocurrent hollow fiber gas separation membranes for process flowsheet synthesis
}

\author{
Bijan Medi ${ }^{1}$ (D) Mobin Nomvar ${ }^{2}$
}

Received: 12 September 2019 / Accepted: 12 February 2020 / Published online: 17 February 2020

(c) Springer Nature Switzerland AG 2020

\begin{abstract}
Membrane separation technology has become an important part of process flowsheet synthesis in which it can be combined with other unit operations for enhanced separation of gasses and liquids. Since process flowsheet synthesis requires computationally efficient numerical algorithms, in this work, an accurate, fast, and robust computer model for the simulation of cocurrent hollow fiber membranes is proposed. The algorithm is based on a modified shooting method with quadratic interpolation, which requires no derivative computations versus classical optimization methods. Moreover, for solving the set of differential equations, three different integration methods were employed and compared over wide range of operating conditions. Based on both speed and robustness, the best method was found to be the variable order numerical differentiation formula as it succeeded in solving the problem fast and accurately under all tested points. The maximum computational time was $3.4 \mathrm{~s}$, while the maximum absolute permeate outlet pressure estimation error was $0.37 \%$, which is remarkable. Also, the method was validated with both experimental and simulation studies in the literature. Hence the proposed algorithm is suitable for process flowsheet synthesis, process design, and optimization studies.
\end{abstract}

Keywords Membrane separation · Numerical solution · Shooting method · Flowsheet synthesis

\section{List of symbols}

A Membrane area, $\mathrm{m}^{2}$

F Molar flow, mol/s

$k$ Number of iterations

$K_{m} \quad$ Permeance, $\mathrm{mol} / \mathrm{m}^{2} \mathrm{~s} \mathrm{~Pa}$

$L \quad$ Membrane length, $\mathrm{m}$

Mw Molecular weight

N Constant defined by Eqs. (20) or (21)

$N_{f} \quad$ Number of hollow fibers

$P$ Pressure, bar

$r$ Hollow fiber radius, $\mu \mathrm{m}$

$T$ Temperature, $\mathrm{K}$

$x \quad$ Spatial coordinate along the hollow fiber length, $\mathrm{m}$

$x_{c} \quad$ Feed-side component mole fraction $y_{c} \quad$ Permeate-side component mole fraction

$z \quad$ Dimensionless spatial coordinate

\section{Greek letters}

$\mu \quad$ Viscosity, Pa s

$\phi \quad$ Parameter defined by Eq. (23)

\section{Subscripts}

c Component/critical

$f \quad$ Feed

in Inlet

o Outer

$p \quad$ Permeate

pex Permeate outlet

$r \quad$ Reduced

$v \quad$ Vapor

Bijan Medi, medi@hut.ac.ir | 1 Department of Chemical Engineering, Hamedan University of Technology, P.O. Box: 65155-579, Hamedan, Iran. ${ }^{2}$ Automated Process Synthesis Co, Sydney, NSW 2044, Australia. 


\section{Introduction}

Process flowsheet synthesis is the computer-aided design of entire plants and processes [1, 2]. It has become the preferred method of process design and will probably play a pivotal role in the analysis of plant economics, safety considerations, environmental issues, and process control.

For a process engineer who has the objective of simulating a single unit operation such as a distillation column, it is quite a rare experience to converge to a reasonable solution in one try without simulation crashes. It is noteworthy that even on commercial software applications, one requires a good level of knowledge to avoid crashes and unrealistic solutions beforehand, while there might be several unit operations on a flowsheet. When it comes to process optimization, where hundreds of alternative solutions are considered, speed, accuracy, and robustness become much more important [3]. Therefore, process simulation for such purposes requires a much higher level of expertise in programming and software development.

Hollow fiber membranes have presented promising solutions in dealing with several important practical separation problems, which aim at global scale issues, including greenhouse emissions and natural gas processing. Their success is supported by rich theoretical and modeling efforts in the past 70 years [4-10]. On the other hand, the membranes can be used in versatile geometrical configurations or be integrated with other separation processes. In general, such problems are defined as flowsheeting for which typically an optimization problem is defined with the freedom on the design of process flowsheet elements.

Hollow fiber membranes are artificial types of semipermeable materials in the form of thousands of bundled annular channels with cross flow of permeates across the walls. The entire module resembles a typical shell and tube heat exchanger. This geometry allows for achieving large packing density, in other words, surface to volume ratio [5].

In most important commercial hollow fiber membrane modules, the feed gas is directed into the shell side at high pressure [5]. The feed constituents tend to pass through the semi-permeable membrane into the annular spaces (permeate side) based on their specific permeances. The ratio of permeabilities is technically called selectivity, which is the most influential parameter quantifying the separation extent [11]. The difference in fugacities or partial pressures, if the components involved can be considered as ideal gases, is the driving force of separation. However, as the components permeate, the pressure varies on both sides of the membrane, and so do fugacities. The pressure variation is larger in the permeate side, and hence it is typically included in the modern membrane models [12].

Another important factor affecting the separation performance is the relative direction of flow of feed and permeate, which can be either cocurrent, counter-current or cross flow. Although the fundamental governing equations of mass, energy, and momentum are similar in the aforementioned modes of operation, they turn out to be different in the numerical solution methods required for computer implementation.

A detailed model of membrane gas separation was first introduced by Weller and Steiner [4] in which they modeled a non-porous membrane for binary separation of gases. Shindo et al. [13] extended the modeling details with more emphasis on the peculiarities of the governing algebraic and differential equations though they did not elaborate on the numerical solution technique. In another important work, Pan [14] presented a practical model for handling high flux asymmetric hollow fiber membranes with the key assumption that back diffusion is negligible from the bulk concentration to the membrane surface. Pan also incorporated the pressure drop in the permeate side, assuming that it follows the well-known Hagen-Poiseuille equation [15].

In another study, Marriott et al. [16] presented the membrane modeling without common assumptions such as plug flow, isothermal operation, steady state conditions, and constant physical properties. They did not present the governing equations, and besides, used commercial software applications for implementation of their suggested model and their extensive studies on the simulation and optimization of numerous membrane systems.

On the other hand, depending on the model type and particular application, there are different types of numerical solution methods, including the iterative shooting method, series (linear) approximations, iterative finite difference, and orthogonal collocation (with and without finite elements). Shindo et al. [13] used the shooting method in their original paper in which the unknown boundary condition is guessed so that the boundary value problem (BVP) is converted into an initial value problem (IVP), but with iterations on the guessed boundary condition. Kovvali et al. [17] postulated that a linear relations exist between the compositions of the feed side and the permeate side, which resulted in an analytical solution of the governing equations for the counter-current mode of operation. Khalilpour et al. [18] converted the cocurrent and counter-current governing differential equations into difference equations using finite difference approximations, and solved the set of nonlinear algebraic equations via the Gauss-Seidel algorithm. 
Orthogonal collocation is a highly accurate numerical method, which is applied to the solution of hollow fiber membrane problems as well as many other systems in the form of boundary value differential equations. Kaldis et al. [19] utilized the Brown's version of this method [20] based on the Pan's original problem formulation, for the cocurrent and counter-current modes of operation and obtained a high level of accuracy. Similarly, the finite element method that shares certain similarities with orthogonal collocation has been applied to membrane simulation independently [21-23] or with the aid of commercial software applications [24]. The main drawback of the orthogonal collocation and finite element methods are complex solution algorithms and massive computational burden in terms of both processing time and memory usage.

Although computational fluid dynamics (CFD) is the most advanced and sophisticated approach toward the simulation of transport phenomena, there are a few studies based on using CFD for simulation of gas separation membrane systems $[25,26]$. The main reason behind this fact is the computational burden, which makes this approach inconvenient for process design and optimization tasks, including flowsheeting applications.

This paper deals with the simulation of gas separation using hollow fiber membrane technology. The general requirements are accuracy, computational time, and robustness against failure. The programming environment is MATLAB as a user-friendly and ubiquitous software package, while compiled $C$ code blocks were used as core programming components for better performance. It has been an objective of this work to keep reliance on the MATLAB toolboxes at a minimum so that code deployment for process flowsheet synthesis on other software applications (e.g., Aspen Plus ${ }^{\mathrm{TM}}$ ) would be easier.

The governing equations which arise in the classical modeling of hollow fiber membranes appear as boundary value problems (BVPs) under the category of ordinary differential equations in the one-dimensional, steady state solution domain. Due to the nature of the problem formulation, and also depending on the solution method, algebraic equations may also arise, but they are always undesirable for the complications they add up to the solution method.

The main problem regarding BVPs is the position of boundary conditions, which do not coincide with the natural direction of the solution method. One classical method of dealing with such problems is the shooting method [27] in which an initial guess is assumed at the required point, and then the problem is iteratively solved to obtain the correct answer at the point with available information. In this way, the entire solution hinges on solving a set of algebraic equations. For each iterative solution, there may not be a realistic corresponding state, and this causes multiple difficulties in the solution approach, including stiffness, divergence, and code crash. Therefore, a multitude of tailored programming techniques is required to make the code fast and robust enough for applications such as process flowsheet synthesis.

In this paper, we introduce a novel numerical solution approach for the simulation of cocurrent hollow fiber gas separation membranes. It is motivated by the market requirement regarding the development of fast, robust, and independent solution methods with minimum reliance on third party computer applications. The paper is summarized as follows: in Sect. 2 and the underlying subsections, first, the governing equations are presented in detail. Then, the numerical methods involved in the programming are described. The main features of the program development are presented and described for a user with a general knowledge of programming. Finally, in Sect. 3, the results are given for validation and test of accuracy, speed, and robustness.

\section{Modeling and simulation}

The key assumptions considered in this work are summarized here, but further elaborated in appropriate places: 1 -The gas is assumed ideal on both sides of the membrane. 2-The process is isothermal, but the temperature effects on gas mixture viscosity and density are accounted. 3-The membrane deformation is negligible. 4-The permeability of the components is constant and hence, it is independent of pressure, temperature, and composition. 5-The pressure change in the feed side is negligible, while the pressure drop in the permeate side follows the Hagen-Poiseuille equation. 6-Axial dispersion is not included in the transport model development.

A hollow fiber membrane is a bundle of annular channels with cross flow of permeates across the channel walls. For such a system, modeling one fiber resembles the entire unit. The problem is schematically depicted in Fig. 1 for the cocurrent arrangement in which the inlet and outlet flows

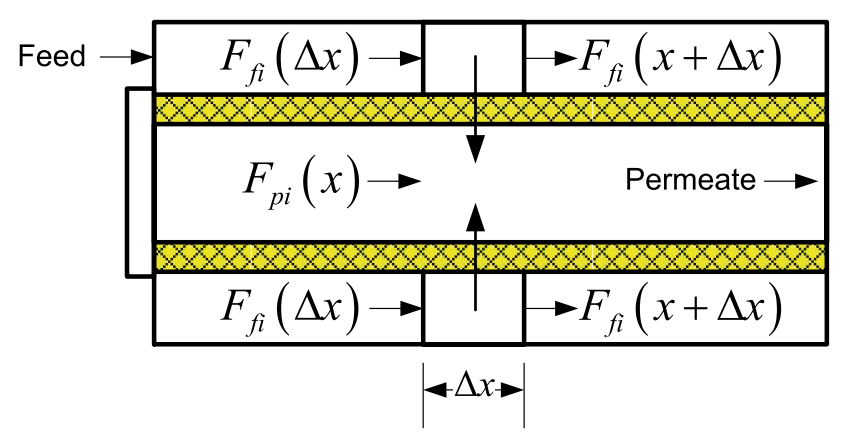

Fig. 1 Problem description for the cocurrent mode of operation 
are shown at the walls of an arbitrary element of length $\Delta x$. Without loss of generality, here we assume that dispersion is negligible along the length of the membrane.

For such a control volume over the feed side, the mass balance with the assumption of no accumulation (i.e., steady state condition) for $N_{f}$ bundled fibers and component molar flow $F_{f}(x)$ can be written as follows:

$0=F_{f}(x)-F_{f}(x+\Delta x)-2 \pi r_{o} N_{f} \Delta x K_{m i}\left(x_{c i} P_{f}-y_{c i} P_{p}\right)$

where $r_{o}$ is the outer radius of hollow fiber, and $K_{m i}, x_{c i}$ and $y_{c i}$ are the permeance, feed-side mole fraction, and permeate-side mole fraction, respectively. $P_{f}$ is the feed-side pressure, which is assumed to be constant, but permeateside pressure $P_{p i}$ varies along the length of the unit.

The trans-membrane mass transfer term $\left(2 \pi r_{o} N_{f} \Delta x K_{m i}\left(x_{c i} P_{f}-y_{c i} P_{p}\right)\right)$ in Eq. (1) is defined based on the difference in partial pressures, which hinges on the ideal gas assumption.

Equation (1) can be rearranged into the formal representation of a difference equation:

$\frac{\Delta F_{f}(x)}{\Delta x}=-2 \pi r_{o} N_{f} K_{m i}\left(x_{c i} P_{f}-y_{c i} P_{p}\right)$

With $\Delta x \rightarrow 0$, Eq. (2) can be written as:

$\frac{d F_{f i}}{d x}=-2 \pi r_{o} N_{f} K_{m i}\left(x_{c i} P_{f}-y_{c i} P_{p}\right)$

It is technically advantageous to normalize this equation, defining $d z=d x / L$ :

$\frac{d F_{f i}}{d z}=-2 \pi r_{o} L N_{f} K_{m i}\left(x_{c i} P_{f}-y_{c i} P_{p}\right)$

The component mole fractions in the feed $\left(x_{c i}\right)$ and the permeate sides $\left(y_{c i}\right)$ can be simply obtained by:

$x_{c i}=\frac{F_{f i}}{\sum_{j=1}^{N_{c}} F_{f j}}$

$y_{c i}=\frac{F_{p i}}{\sum_{j=1}^{N_{c}} F_{p j}}$

Although it is possible to establish similar differential equations for the permeate side, a simple mass balance can be used instead to obtain component molar flows:

$F_{p i}=F_{f i 0}-F_{f i}$

A similar equation holds for the overall mass balance in the permeate side:

$F_{p}=F_{f 0}-F_{f}$
Note that for $N_{C}$ components, Eq. (4) gives rise to a set of $N_{C}$ coupled nonlinear initial value differential equations. The boundary conditions for this set of IVPs for both cocurrent and counter-current arrangements are given for the inlet side of the feed flow:

$F_{f}(z=0)=F_{f 0}$

$x_{c}(z=0)=x_{c 0}$

noting that $F_{f i}=x_{c i} F_{f}$.

It must be emphasized that our formulation has the minimum number of algebraic equations, which is beneficial in terms of code robustness and matrix operations.

The pressure variations along the membrane unit must be either specified or calculated properly. It is common to assume constant pressure along the feed side of the unit. However, the pressure drop along the permeate side is typically calculated via the well-known Hagen-Poiseuille equation, which is given hear for an ideal gas at temperature $T$ flowing in a long cylindrical pipe with constant cross section $[12,18]$ :

$\frac{d P_{p}}{d z}=-\frac{8 R T L \mu_{m} F_{p}}{\pi r_{i n}^{4} N_{f} P_{p}}$

$r_{i n}, \mu_{m}$ are hollow fiber inner radius and mixture viscosity, respectively.

For cocurrent flow, the pressure is usually known at the outlet condition (downstream pressure):

$P_{p}(z=1)=P_{p e x}$

This boundary condition transforms the problem into a BVP one, which must be solved iteratively, for example by the shooting method, which is described in Sect. 2.2.1.

It is noteworthy that the composition of the permeate side at $z=0$ must be calculated based on the consideration of zero permeate flow rate $\left(F_{p i}=0\right)$ as suggested by Shindo et al. [13]:

$y_{c i}=\frac{K_{m i}\left(x_{c i} P_{f}-y_{c i} P_{p}\right)}{\sum_{i=1}^{N_{c}}\left(x_{c i} P_{f}-y_{c i} P_{p}\right)}$

For multi-component separations, it is necessary to solve this equation by trial and error. We have used the Gauss-Seidel iterative algorithm though we did not find any significant difference in the entire solution by setting all $y_{c i}$ values to zero at $z=0$.

\subsection{Thermophysical properties}

In this study, the physical and thermodynamic properties are treated with several simplifying assumptions; the gas is considered ideal on both sides of the membrane unit. In 
particular, it is included in the derivation of the Hagen-Poiseuille equation. Furthermore, the gas mixture viscosity is required for the pressure drop calculation, which is elaborated in the "Appendix".

\subsection{Numerical methods}

Regardless of the type of boundary value problem, the core of the solution technique is based on the solution of a set of ordinary differential equations (ODEs). There are numerous algorithms for solving any specific system depending on the type of boundary conditions, nonlinearity, size of the problem, stiffness, etc. The current problem is prone to stiffness, which is described as the conditions in which variations in one or more differential variables occur in vastly different scales. In such conditions, a too large solution step reduces the solution accuracy, while a too small solution step increases the computational time, and taxes on the computer processing and memory resources. Hence we focus on some of the most efficient generalpurpose and proprietary integration formulas for handling stiff problems in a comparative manner.

In MATLAB, ode45 which is based on an explicit Runge-Kutta $(4,5)$ formula, the Dormand-Prince pair [28], is the most accurate solver and usually the first choice for most problems. In the following, this algorithm is designated as RK45 for brevity. For stiff problems, other methods may be beneficial. The ode15s algorithm in MATLAB, which is based on numerical differentiation formulas (NDFs) of order 1-5 [29,30], is recommended [31]. Optionally, it also uses the backward differentiation formulas (BDFs, also known as the Gear's method).

We have also utilized the modified extended backward differentiation formula (MEBDF), which has been developed by Cash [32] for the numerical solution of stiff initial value problems in the form of ODEs and differential-algebraic equations (DEAs). This method has been originally proposed as a class of variable order, variable step boundary value formulas.

\subsubsection{The shooting method}

As pointed earlier, the solution of the set of differential equations at hand requires iterative procedures since the boundary conditions do not occur in one location along the solution domain. A classical method of solving such problems is the shooting method [27], in which a set of initial guesses is given for the unknown boundary conditions at appropriate locations (in time or space). Then the solution is iterated by comparing the resulting boundary conditions from solving the set of ODEs until the difference becomes insignificant with respect to a given tolerance.
The generally nonlinear problem can be formulated in several iterative ways. We have chosen an error minimization approach by defining an objective function as:

$f\left(x^{k}\right)=\left|\frac{B C(k)-B C(k-1)}{B C(k-1)}\right|$

where $B C$ is the unknown boundary condition and $k$ is the number of iterations.

Minimization of this function was first carried out using MATLAB Optimization Toolbox, which offers a variety of constrained and unconstrained solution techniques such as SQP, Active-Set, Interior Point, and even single-objective Genetic Algorithm (GA) [33]. However, since none of the methods was efficient enough for this problem, and also we were interested not to excessively rely on MATLAB proprietary toolboxes, we adopted the quadratic interpolation technique [3], which is a simple but efficient algorithm for univariate optimization problems (single decision variable, single objective function). It is based on the assumption that the objective function $f$ can be approximated by a quadratic function in a local neighborhood:

$f(x)=a+b x+c x^{2}$

Upon taking the first derivative, it is easy to prove that this function has an extremum (minimum or maximum) at:

$x^{*}=-\frac{b}{2 c}$

For $c>0, x^{*}$ is a minimum, and for $c<0$, it is a maximum. For continuous functions, this method guarantees a local optimum, while it requires neither analytical nor numerical derivatives of any order of the objective function. Besides, it needs only one function call in each iteration (except the first iteration in which it requires three), which makes it the best algorithm in this regard. In fact, the only drawback of this algorithm is that it can be used only for univariate problems.

The implementation of the quadratic interpolation method is described in the following steps:

1. First and probably the most challenging step is finding three initial points $\left(x_{1}, x_{2}\right.$, and $\left.x_{3}\right)$ for the unknown $B C$ in such a way that their respective function values $\left(f_{1}, f_{2}\right.$, and $f_{3}$ ) form a valley as shown in Fig. 2 . For continuous functions, this step guarantees that at least one minimum will be found in the area confined in this valley.

Having found $f_{1}, f_{2}$, and $f_{3}$, the new point $\left(\tilde{x}^{*}\right)$ is calculated from the following formula [3]:

$$
\tilde{x}^{*}=\frac{1}{2}\left[\frac{\left(x_{2}^{2}-x_{3}^{2}\right) f_{1}+\left(x_{3}^{2}-x_{1}^{2}\right) f_{2}+\left(x_{1}^{2}-x_{2}^{2}\right) f_{3}}{\left(x_{2}-x_{3}\right) f_{1}+\left(x_{3}-x_{1}\right) f_{2}+\left(x_{1}-x_{2}\right) f_{3}}\right]
$$




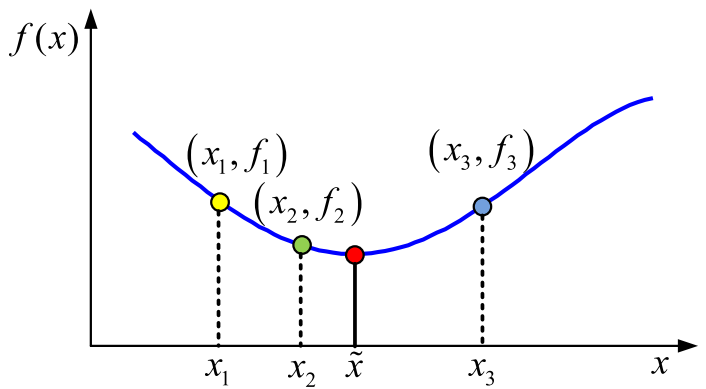

Fig. 2 Schematic representation of encompassing a local extremum (minimum) in the quadratic interpolation method

2. $x_{1}, x_{2}, x_{3}$, and $\tilde{x}^{*}$ are first sorted based on their values. Then, the minimum point $\left(\tilde{x}^{*}\right)$ along with its two neighbors are selected for the next level of optimization. In this way, the three points gradually approach a local minimum.

3. The termination criterion is the satisfaction of both of the following conditions:

$\frac{\left|x_{1}-x_{3}\right|}{\left|x_{1}\right|+\varepsilon} \leq \operatorname{TolX}$

$f\left(x^{*}\right) \leq$ TolF

TolX and TolF are two arbitrary small values $\left(10^{-3}\right.$ and $10^{-2}$, respectively), and $\varepsilon$ is a very small value $\left(\sim 10^{-16}\right)$, which avoids division by zero. The algorithm is illustrated in Fig. 3.

As mentioned earlier, it is not easy to find three initial points such that their respective objective function values form a valley. It must be noted that for too small inlet permeate pressures $\left(P_{p}\right.$ at $\left.z=0\right)$, the problem becomes stiff, even forcing the ODE solver to quit before integration reaches the end of the domain $(z=1)$. For this case, an artificial penalty is assigned to the value of $f$. On the other hand, for very large inlet permeate pressures, the mass flux might be reversed and causes negative permeate flow rates, which is physically infeasible. Hence, the upper limit is decided by the user, while a value around $P_{f 0} / 2$ can be recommended for most problems to start with.

For the lower limit, the first guess of 1 bar is appropriate for most problems. Then the middle point is guessed by, for example, using the average value of lower and upper points. If these three points do not satisfy the valley condition, the interval is reduced by successively squeezing the lower and upper limits with a moving average for the middle point. In each iteration, we reduce the upper and increase the lower limits of permeate pressure guesses by $10 \%$ and $5 \%$, respectively.

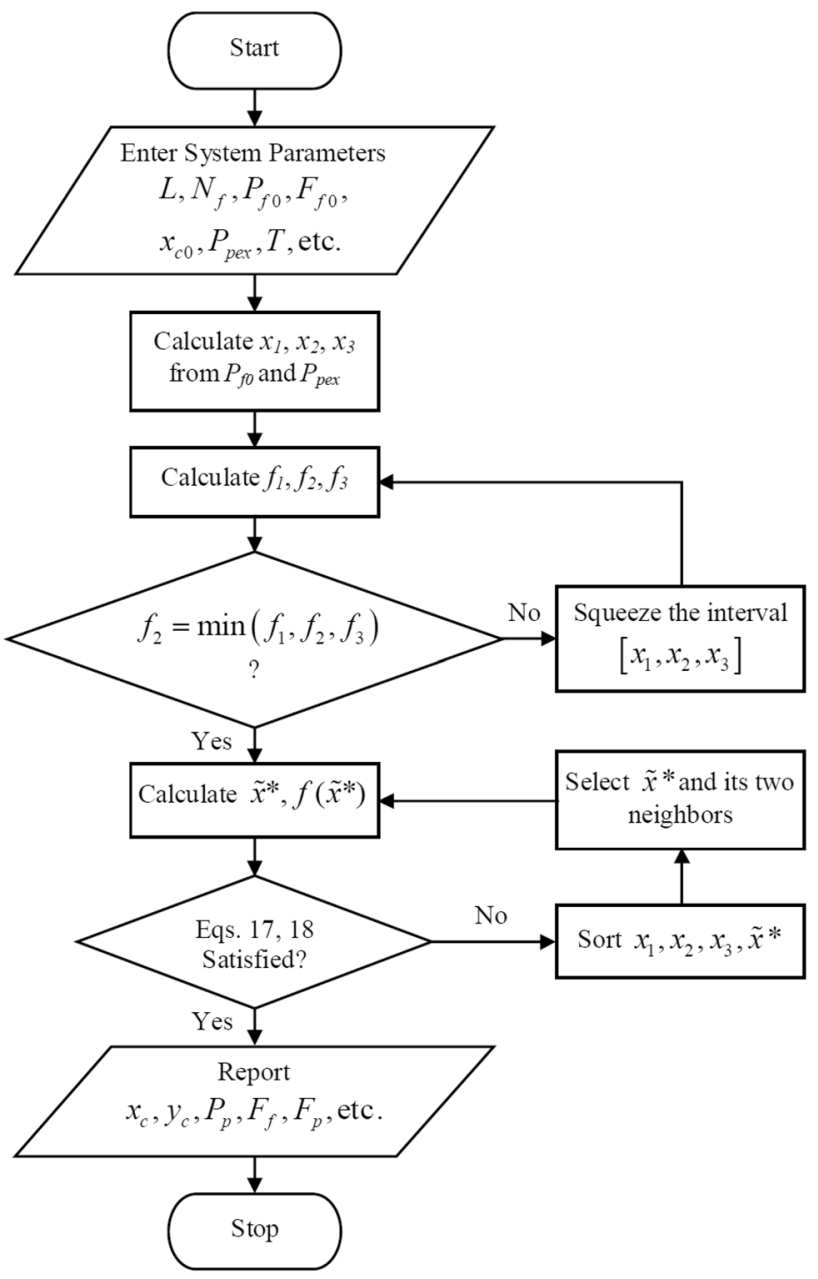

Fig. 3 The flowchart of the modified shooting method

\subsection{Programming}

MATLAB is a popular environment for writing general-purpose programs. It is still among the top-ten programming languages [34], and appears to be more prosperous as it expands its connections with other software and hardware products. For all the simulations, MATLAB 2014a on a laptop with an Intel Core i5-2430 M (2.40 GHz) CPU with 6 GB RAM is used. The differential equations and the objective function for the shooting method were written in the $C$ programming language and compiled for application within MATLAB. The codes are available for test and use upon request.

\section{Results}

The results are given in two parts; in the first part, the numerical methods are validated with available experimental data and also simulation profiles. In the second 


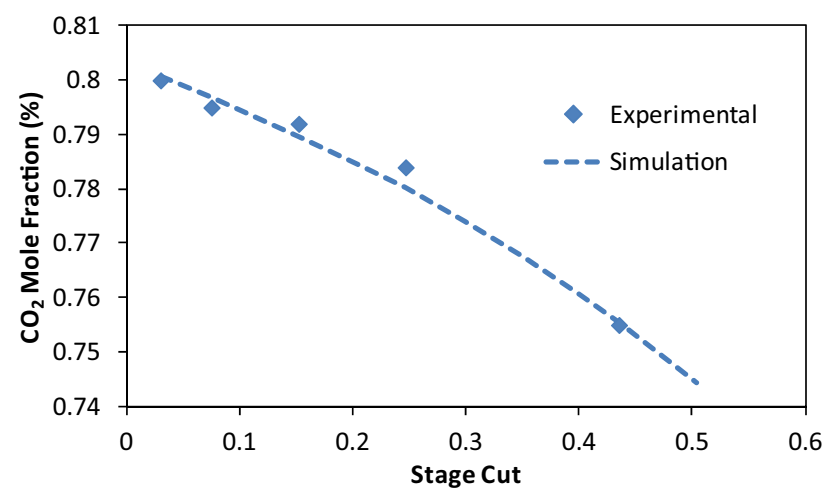

Fig. 4 Comparison of the $\mathrm{CO}_{2}$ mol fraction in the permeate side from the current work with the experimental data of Tranchino et al. [35]. The feed is $60 \% \mathrm{CO}_{2}$ and $40 \% \mathrm{CH}_{4}$

Table 1 System and operating parameters used for validation by the experimental data of Tranchino et al. [35]

\begin{tabular}{lll}
\hline Parameter & Unit & Value \\
\hline Temperature $(T)$ & ${ }^{\circ} \mathrm{C}$ & 25 \\
Feed inlet pressure $\left(P_{f 0}\right)$ & bar & 4.053 \\
Permeate outlet pressure $\left(P_{\text {pex }}\right)$ & bar & 1.013 \\
Length of unit $(L)$ & $\mathrm{m}$ & 0.15 \\
Fiber inner radius $\left(r_{i n}\right)$ & $\mu \mathrm{m}$ & 389 \\
Fiber outer radius $\left(r_{o}\right)$ & $\mu \mathrm{m}$ & 725 \\
Permeance of $\mathrm{CO}_{2}$ & $\mathrm{~mol} / \mathrm{m}^{2} \mathrm{~s} \mathrm{~Pa}$ & $3.16 \times 10^{-9}$ \\
Permeance of $\mathrm{CH}_{4}$ & $\mathrm{~mol} / \mathrm{m}^{2} \mathrm{~s} \mathrm{~Pa}$ & $8.81 \times 10^{-10}$ \\
\hline
\end{tabular}

part, the integration methods are compared in terms of computational time and permeate outlet pressure estimation error. For all the integration methods, the absolute and relative tolerances are $10^{-6}$ and $10^{-4}$, respectively.

\subsection{Validation}

The simulation of the hollow fiber gas separation membrane in the cocurrent mode is first validated via comparison with the experimental results of Tranchino et al. [35], which were given for the separation of a mixture of $\mathrm{CO}_{2}$ and $\mathrm{CH}_{4}$ in a laboratory setup. As can be seen in Fig. 4, in a wide range of stage cuts obtained by changing the feed flow rate, the suggested numerical method offers acceptable accuracy with respect to the experimental data. The system and operating conditions are given in Table 1. The feed is $60 \% \mathrm{CO}_{2}$ and $40 \% \mathrm{CH}_{4}$.

Moreover, the pressure and composition profiles along the hollow fiber membrane are compared with the simulation results given in Khalilpour et al. [18]. Note that they have validated their codes with some experimental results in the countercurrent mode of operation. The operating
Table 2 Operating parameters used for validation by simulation work of Khalilpour et al. [18]

\begin{tabular}{lll}
\hline Parameter & Unit & Value \\
\hline Temperature $(T)$ & ${ }^{\circ} \mathrm{C}$ & 25 \\
Feed inlet pressure $\left(P_{f 0}\right)$ & bar & 30 \\
Permeate outlet pressure $\left(P_{p e}\right)$ & bar & 1.013 \\
Length of unit $(L)$ & $\mathrm{m}$ & 1 \\
Fiber inner radius $\left(r_{\text {in }}\right)$ & $\mu \mathrm{m}$ & 40 \\
Fiber outer radius $\left(r_{o}\right)$ & $\mu \mathrm{m}$ & 100 \\
Membrane area $(A)$ & $\mathrm{m}^{2}$ & 25 \\
Total feed flow rate $\left(F_{f 0}\right)$ & $\mathrm{mol} / \mathrm{s}$ & 1 \\
\hline
\end{tabular}

Table 3 Feed composition and permeances of the components used for validation by simulation work of Khalilpour et al. [18]

\begin{tabular}{lcc}
\hline Parameter & Composition (\%) & $\begin{array}{l}\text { Permeance } \\
\left(\mathrm{mol} / \mathrm{m}^{2} \mathrm{sPa}\right)\end{array}$ \\
\hline $\mathrm{CO}_{2}$ & 20.0 & $1.34 \times 10^{-8}$ \\
$\mathrm{CH}_{4}$ & 60.0 & $3.72 \times 10^{-10}$ \\
$\mathrm{C}_{2} \mathrm{H}_{6}$ & 15.0 & $1.02 \times 10^{-10}$ \\
$\mathrm{C}_{3} \mathrm{H}_{8}$ & 5.0 & $0.2 \times 10^{-10}$ \\
\hline
\end{tabular}

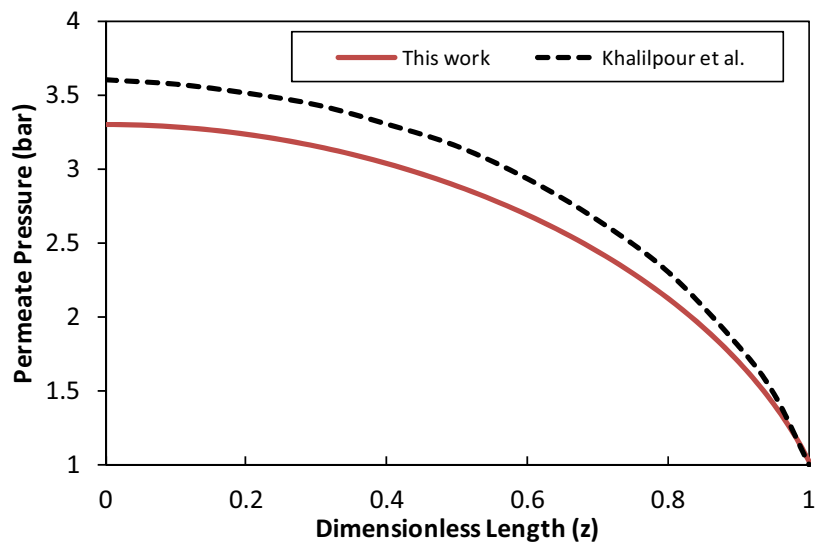

Fig. 5 Comparison of the pressure profile from the current work with Khalilpour et al. [18]

conditions are given in Table 2. The feed composition and permeance values are given in Table 3.

The simulated permeate pressure profile along the length of the unit is given in Fig. 5 for this work and the reference simulation [18]. The difference is maximum at the inlet side $(\sim 8.5 \%)$, and it decreases toward the outlet side. It is necessary to mention that when the mixture viscosity calculated by Eq. (22) was replaced with air viscosity at the feed inlet conditions, the difference became much smaller, suggesting that probably the air viscosity was used in the reference simulation. It is also 


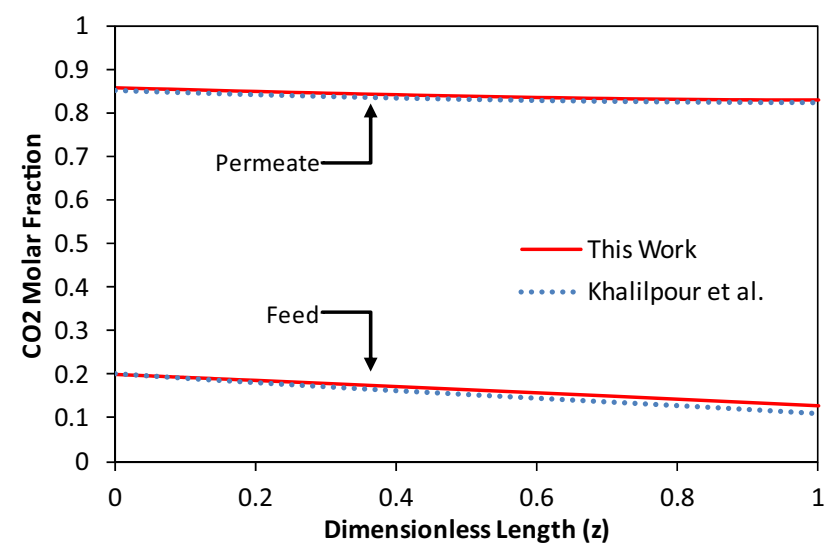

Fig. 6 Comparison of the $\mathrm{CO}_{2}$ mol fraction profiles in the feed and permeate sides with Khalilpour et al. [18]

necessary to mention that the calculated mixture viscosity was checked with the results of Aspen Plus ${ }^{\mathrm{TM}}$ software for which the differences were insignificant.

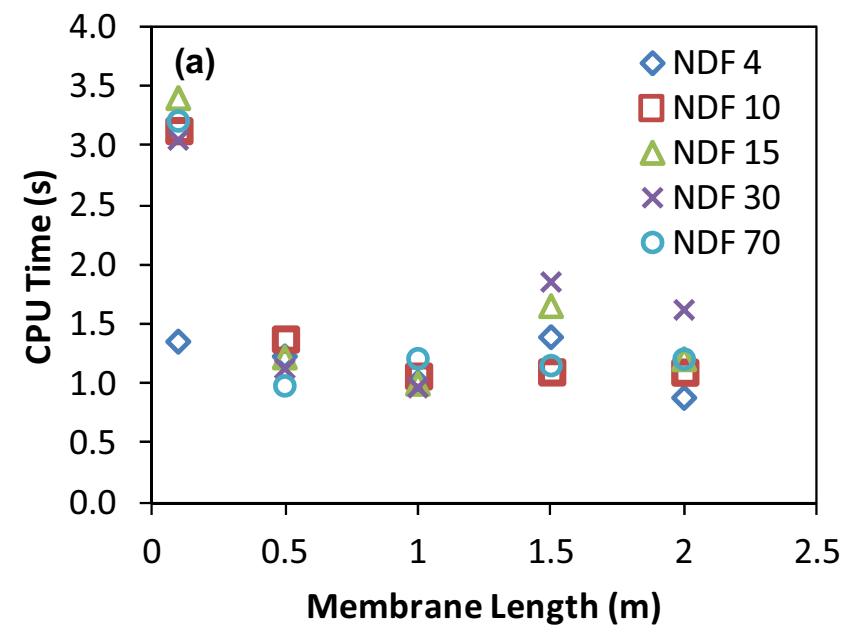

The $\mathrm{CO}_{2}$ mol fractions in the feed and permeate sides are given in Fig. 6 and compared with the simulation results of Khalilpour et al. [18]. Here also, the differences are small compared to the reference simulation. The difference in the permeate side is less than $1.0 \%$, which occurs at the closed end of the hollow fiber. The difference is larger in the feed side with the maximum at the exit $(\sim 13.2 \%)$, but here again, using air viscosity, it becomes smaller.

\subsection{Parametric study}

In this section, the performance of the numerical methods under study is tested against changes in the hollow fiber membrane length and feed pressure as two important design/operating parameters. The ranges of variations are selected according to the practical values reported in the literature. Other parameters are fixed at those of the simulation results given earlier.

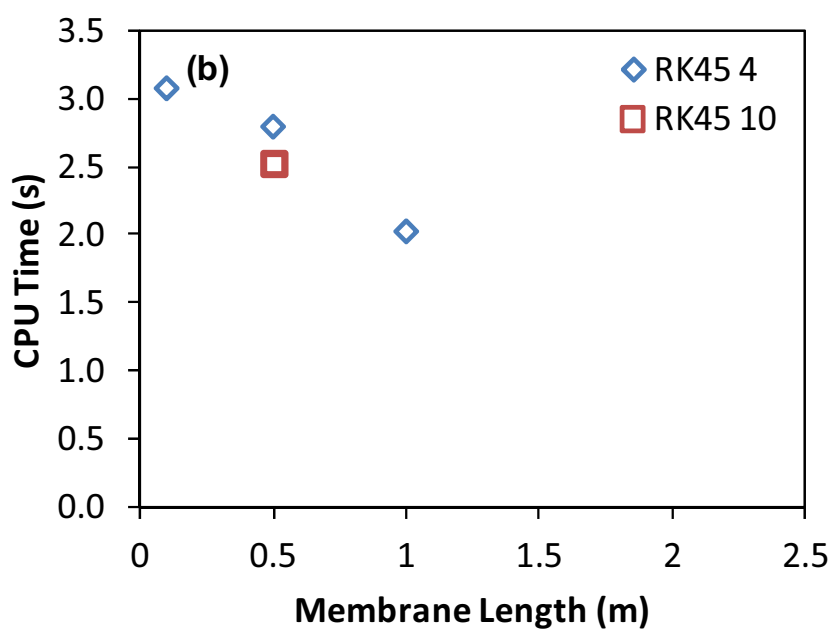

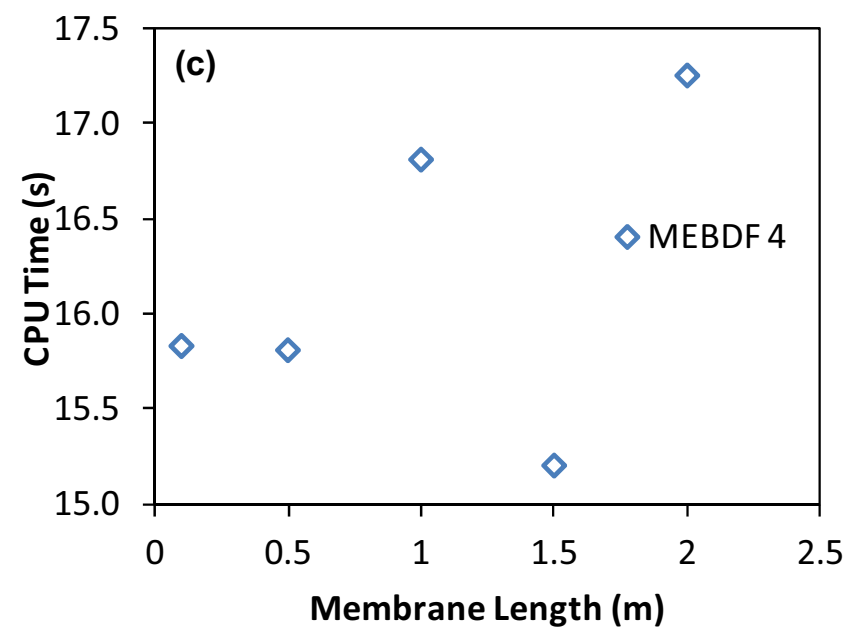

Fig. 7 Computational time (in seconds) for a NDF, b RK45, and c MEBDF algorithms. The values in legends designate feed pressure (in bar) 

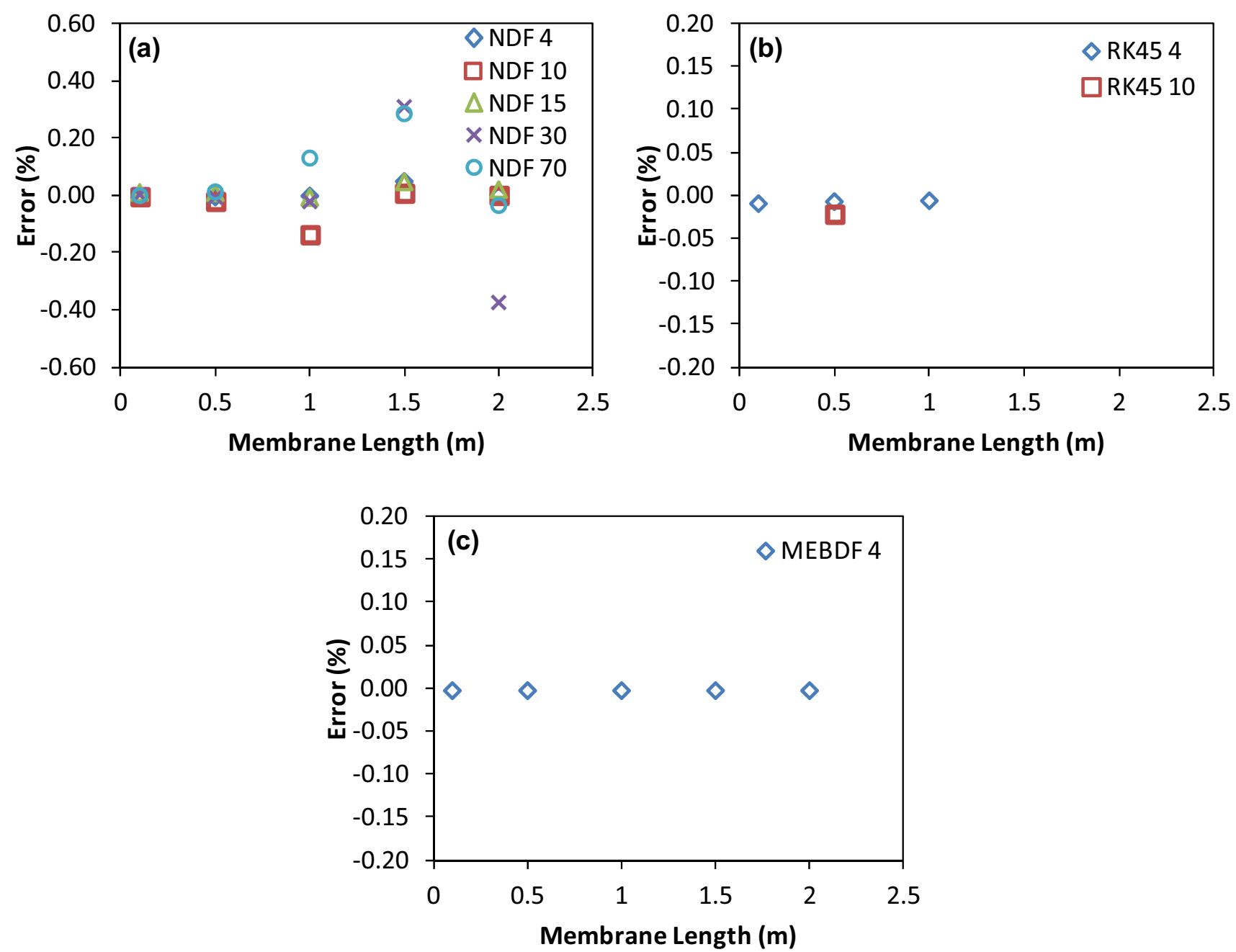

Fig. 8 Error in outlet permeate pressure $\left(P_{\text {pex }}\right)$ calculations (in percents) using the shooting method with a NDF, b RK45, and c MEBDF algorithms. The values in the legends designate feed pressure (in bar)

The computational times are compared in Fig. 7. The plotted points refer to the runs that the algorithms succeeded in finding a solution in a finite time. As can be seen, not only the NDF algorithm succeeds in all tested conditions, it also offers the minimum computational time. On the other hand, the RK45 and the MEBDF have failed in most of the tested points.

Similarly, permeate outlet pressure calculation errors are given in Fig. 8 in percents. While the NDF offers very small error values, the only advantage of the MEBDF can be seen here as the error values for this method are smaller compared to the other methods.

\section{Conclusion}

There are two important requirements for any computer code that is intended for process flowsheet synthesis applications: robustness and speed. It is a matter of disgrace for any simulation software if the code crashes or the system freezes while processing a simulation case. In this work, we applied a straightforward method for solving the differential-algebraic equations, which arise in modeling cocurrent hollow fiber membranes for gas separation. The NDF method employed for solving the differential equations offers acceptable accuracy with maximum robustness under all the tested conditions. 
Regarding the algebraic equation that arises from solving the boundary value problem using the shooting method, the quadratic interpolation method was adjusted to offer fast and reliable results. It must be emphasized that this method does not employ matrix operations, nor does it require differentiation of any kind, which are important advantages. The main difficulty in this method however, is finding three initial guesses properly, which was handled in this work with a search area squeezing policy.

While the proposed method was tailored for hollow fiber membrane simulation in the context of process flowsheet synthesis, with proper adjustments, it can be applied to other similar systems of boundary value problems. However, it must be asserted that for very high pressure systems such as natural gas processing, the non-ideal behaviors (e.g., deviations from ideal gas, polarization, and non-isothermal effects) must be taken into account for hollow fiber membrane modeling and simulation.

Funding This research did not receive any specific grant from funding agencies in the public, commercial, or not-for-profit sectors.

\section{Compliance with ethical standards}

Conflict of interest The authors declare that they have no conflict of interest.

\section{Appendix}

Here the equations required for the calculation of vapor viscosity of gaseous mixtures are given. In general, such relations are semi-empirical and subject to limited species and specified pressures and temperatures. We have used the method of Stiel and Thodos [36] for the prediction of vapor viscosity of pure hydrocarbons at low pressure (below reduced temperature $\left(T_{r}\right)$ of 0.6 ) as reported in the Perry's handbook as the most accurate method [37]. Only molecular weight $\left(M w_{i}\right)$, critical temperature $\left(T_{c i}\right)$, and critical pressure $\left(P_{c i}\right)$ are required:

$\mu_{v i}=\frac{4.60 \times 10^{-4} N M w_{i}^{1 / 2} P_{c i}^{2 / 3}}{T_{c i}^{1 / 6}}$

$N=0.0003400 T_{r i}^{0.94}$ for $T_{r i} \leq 1.5$

$N=0.0001778\left(4.58 T_{r i}-1.67\right)^{0.625}$ for $T_{r i}>1.5$

The viscosity in Eq. (19) is in centipoise ( $\mathrm{mPa}$ ) if $T_{c}$ and $P_{c}$ are given in $\mathrm{K}$ and $\mathrm{Pa}$, respectively. This method can also be used for light non-hydrocarbon gases except for hydrogen, where special formulations are required to calculate $N$. For hydrocarbons below ten carbon atoms, average errors of about 3\% are expected, with errors increasing to $5-10 \%$ for heavier hydrocarbons [37].

For the prediction of the vapor viscosity of gaseous mixtures of hydrocarbon and nonhydrocarbon gases at low pressures below a $T_{r}$ of 0.6 , the method of Bromley and Wilke [38] is recommended. However, the equations given in the Perry's handbook [37] do not seem to be correct. Therefore, the equations are reported from the original Ref. [38]:

$\mu_{m}=\sum_{i=1}^{N_{c}} \frac{\mu_{v i}}{1+\frac{1}{y_{c i}} \sum_{\substack{j=1 \\ j \neq i}}^{N_{c}} y_{c j} \phi_{i j}}$

where:

$\phi_{i j}=\frac{\left[1+\left(\frac{\mu_{v i}}{\mu_{v j}}\right)^{1 / 2}\left(\frac{M w_{j}}{M w_{i}}\right)^{1 / 4}\right]^{2}}{\sqrt{8}\left[1+\frac{M w_{i}}{M w_{j}}\right]^{1 / 2}}$

\section{References}

1. Bommareddy S, Eden MR, Gani R (2011) Computer aided flowsheet design using group contribution methods. In: Pistikopoulos EN, Georgiadis MC, Kokossis AC (eds) Computer aided chemical engineering, vol 29. Elsevier, Amsterdam, pp 321-325. https://doi.org/10.1016/B978-0-444-53711-9.50065-1

2. Shafiee A, Nomvar M, Liu Z, Abbas A (2017) Automated process synthesis for optimal flowsheet design of a hybrid membrane cryogenic carbon capture process. J Clean Prod 150:309-323. https://doi.org/10.1016/j.jclepro.2017.02.151

3. Edgar TF, Himmelblau DM, Lasdon LS (2001) Optimization of chemical processes. McGraw-Hill chemical engineering series, 2nd edn. McGraw-Hill, New York

4. Weller S, Steiner WA (1950) Separation of gases by fractional permeation through membranes. J Appl Phys 21(4):279-283. https://doi.org/10.1063/1.1699653

5. Ahmad F, Lau KK, Lock SSM, Rafiq S, Khan AU, Lee M (2015) Hollow fiber membrane model for gas separation: process simulation, experimental validation and module characteristics study. J Ind Eng Chem 21:1246-1257. https://doi.org/10.1016/j. jiec.2014.05.041

6. Chowdhury MHM (2012) Simulation, design and optimization of membrane gas separation, chemical absorption and hybrid processes for $\mathrm{CO}_{2}$ capture. UWSpace, Madison

7. Heydari S, Pirouzfar V (2016) The influence of synthesis parameters on the gas selectivity and permeability of carbon membranes: empirical modeling and process optimization using surface methodology. RSC Adv 6(17):14149-14163. https://doi. org/10.1039/c5ra27772h

8. Pirouzfar V, Hosseini SS, Omidkhah MR, Moghaddam AZ (2014) Modeling and optimization of gas transport characteristics of carbon molecular sieve membranes through statistical analysis. Polym Eng Sci 54(1):147-157. https://doi.org/10.1002/ pen.23553 
9. Pirouzfar V, Omidkhah MR (2016) Mathematical modeling and optimization of gas transport through carbon molecular sieve membrane and determining the model parameters using genetic algorithm. Iran Polym J 25(3):203-212. https://doi. org/10.1007/s13726-016-0414-z

10. Tessendorf S, Gani R, Michelsen ML (1999) Modeling, simulation and optimization of membrane-based gas separation systems. Chem Eng Sci 54(7):943-955. https://doi.org/10.1016/S0009 -2509(98)00313-3

11. Hoorfar M, Alcheikhhamdon $Y$, Chen B (2018) A novel tool for the modeling, simulation and costing of membrane based gas separation processes using Aspen HYSYS: optimization of the $\mathrm{CO}_{2} / \mathrm{CH}_{4}$ separation process. Comput Chem Eng 117:11-24. https://doi.org/10.1016/j.compchemeng.2018.05.013

12. Hosseini SS, Roodashti SM, Kundu PK, Tan NR (2015) Transport properties of asymmetric hollow fiber membrane permeators for practical applications: mathematical modelling for binary gas mixtures. Can J Chem Eng 93(7):1275-1287. https://doi. org/10.1002/cjce.22215

13. Shindo Y, Hakuta T, Yoshitome H, Inoue H (1985) Calculation methods for multicomponent gas separation by permeation. Sep Sci Technol 20(5-6):445-459

14. Pan CY (1986) Gas separation by high-flux, asymmetric hollow-fiber membrane. AIChE J 32(12):2020-2027. https://doi. org/10.1002/aic.690321212

15. Berman AS (1953) Laminar flow in channels with porous walls. J Appl Phys 24(9):1232-1235. https://doi.org/10.1063/1.1721476

16. Marriott JI, Sørensen E, Bogle IDL (2000) Detailed mathematical modelling of membrane modules. In: Pierucci S (ed) Computer aided chemical engineering, vol 8. Elsevier, Amsterdam, pp 523-528. https://doi.org/10.1016/S1570-7946(00)80089-9

17. Kovvali AS, Vemury S, Admassu W (1994) Modeling of multicomponent countercurrent gas permeators. Ind Eng Chem Res 33(4):896-903. https://doi.org/10.1021/ie00028a016

18. Khalilpour R, Abbas A, Lai Z, Pinnau I (2013) Analysis of hollow fibre membrane systems for multicomponent gas separation. Chem Eng Res Des 91(2):332-347. https://doi.org/10.1016/j. cherd.2012.07.009

19. Kaldis SP, Kapantaidakis GC, Sakellaropoulos GP (2000) Simulation of multicomponent gas separation in a hollow fiber membrane by orthogonal collocation-hydrogen recovery from refinery gases. J Membr Sci 173(1):61-71. https://doi. org/10.1016/S0376-7388(00)00353-7

20. Brown KM, Conte SD (1967) The solution of simultaneous nonlinear equations. Paper presented at the Proceedings of the 1967 22nd national conference, Washington, DC, USA

21. Dehkordi Javad A, Hosseini Seyed S, Kundu Prodip K, Tan Nicolas $R$ (2016) Mathematical modeling of natural gas separation using hollow fiber membrane modules by application of finite element method through statistical analysis. Chem Product Process Model. https://doi.org/10.1515/cppm-2015-0052

22. Dehkordi JA, Hosseini SS, Kundu PK, Tan NR (2015) Mathematical modeling of natural gas separation using hollow fiber membrane modules by application of finite element method through statistical analysis. Chem Prod Process Model 11(1):11-15
23. Hosseini SS, Dehkordi JA, Kundu PK (2016) Gas permeation and separation in asymmetric hollow fiber membrane permeators: mathematical modeling, sensitivity analysis and optimization. Korean J Chem Eng 33(11):3085-3101. https://doi.org/10.1007/ s11814-016-0198-z

24. Nosratinia F, Ghadiri M, Ghahremani H (2014) Mathematical modeling and numerical simulation of ammonia removal from wastewaters using membrane contactors. J Ind Eng Chem 20(5):2958-2963. https://doi.org/10.1016/j.jiec.2013.10.065

25. Harasek M, Haddadi B, Miltner M, Schretter P, Jordan C (2016) Fully resolved CFD simulation of a hollow fibre membrane module. Chem Eng Trans 52:433-438

26. Haddadi B, Jordan C, Schretter P, Lassmann T, Harasek M (2016) Designing better membrane modules using CFD. Chem Prod Process Model 11:57-66

27. Pardyjak E (2018) Boundary value problems, chapter 27. http:// www.mech.utah.edu/ pardyjak/me6700/Lect15_BoundEigen valueProblemsCh27.pdf

28. Dormand JR, Prince PJ (1980) A family of embedded RungeKutta formulae. J Comput Appl Math 6(1):19-26. https://doi. org/10.1016/0771-050X(80)90013-3

29. Shampine LF, Reichelt MW (1997) The MATLAB ODE suite. SIAM J Sci Comput 18:1-22

30. Shampine LF, Reichelt MW, Kierzenka JA (1999) Solving index-1 DAEs in MATLAB and simulink. SIAM Rev 41:538-552

31. Mathworks MATLAB Documentation Center: choosing an ODE solver. https://www.mathworks.com/help/matlab/math/choos e-an-ode-solver.html. Accessed 15 Aug 2019

32. Cash JR (2000) Modified extended backward differentiation formulae for the numerical solution of stiff initial value problems in ODEs and DAEs. J Comput Appl Math 125(1):117-130. https ://doi.org/10.1016/S0377-0427(00)00463-5

33. Mathworks (2014) Global optimization toolbox 3.2.5 user guide

34. TIOBE (2018) TIOBE Index for October 2018. https://www.tiobe .com/tiobe-index/. Accessed 22 Oct 2018

35. Tranchino L, Santarossa R, Carta F, Fabiani C, Bimbi L (1989) Gas separation in a membrane unit: experimental results and theoretical predictions. Sep Sci Technol 24(14):1207-1226. https:// doi.org/10.1080/01496398908049898

36. Stiel LI, Thodos $\mathrm{G}$ (1961) The viscosity of nonpolar gases at normal pressures. AIChE J 7(4):611-615. https://doi.org/10.1002/ aic. 690070416

37. Liley PE, Thomson GH, Friend DG, Daubert TE, Buck E (1997) Physical and chemical DATA. In: Perry RH, Green DW, Maloney JO (eds) Perry's chemical engineers' handbook, 7th edn. McGrawHill, New York, p 373

38. Bromley LA, Wilke CR (1951) Viscosity behavior of gases. Ind Eng Chem 43(7):1641-1648. https://doi.org/10.1021/ie50499a046

Publisher's Note Springer Nature remains neutral with regard to jurisdictional claims in published maps and institutional affiliations. 